\title{
Editorial
}

\section{Clinical Insights: Biomechanics and lameness diagnosis}

The gold standard for accurate lameness evaluation in the horse has been extensively discussed in two of our recent editorials [1,2]. This debate centres around whether the gold standard should be the experienced clinician or objective gait data. Veterinary professionals are divided on whether quantitative gait analysis is or it is not a valuable clinical tool; and around the concepts of lameness and locomotion asymmetry. The conclusions drawn so far are: a lame horse should be one deemed unfit to be used for ridden exercise, other sports or activities based on a comprehensive evaluation; lameness has a negative emotional value in society; lameness does not equal to gait asymmetry, but asymmetry may indicate lameness in the presence or absence of pain; subtle lameness is a grey area where gait asymmetry may fall either inside or outside the threshold set for quantitative gait analysis, quantitative gait analysis can provide such asymmetry measurements, of either limb loadings or upper body and limbs motion; clinicians must decide on the clinical relevance of a measured locomotion asymmetry using stablished reference values; and finally, that technology does not aim to replace clinical judgment but to aid it.

In the meantime, veterinary clinics, university hospitals and individual vets worldwide are increasingly acquiring equipment for objective lameness quantification, mainly inertial measurement units (IMUs), but also foot pressure analysis systems, to complement and/or confirm their clinical diagnosis, in a similar fashion as they might use diagnostic imaging. More and more equine clinicians and vet students around the globe have access to the latest research on the biomechanics of lameness, thus familiarising themselves with the complex mechanics of lameness compensation and the latest technology to quantify it. Therefore, it is imperative to provide proper educational material to fulfil the needs of those veterinarians eager to learn and apply such technologies, and to keep them up-todate with the latest investigations on the biomechanics of normal locomotion and lameness and the use of quantitative gait analysis tools for diagnosis of lameness.

Gait disturbances are not exclusive to the musculoskeletal system and can also have neurological origin, therefore gait data analysis can be also applied to neurological cases. There is poor agreement between observers making subjective assessments of equine neurological gait abnormalities, as there is with visual examination of non-neurological lameness. A study aiming to stimulate a dosedependent ataxia in horses using xylazine resulted in significant changes of gait parameters, pelvic accelerations, and stabilographic variables (such as the development of rostrocaudal and mediolateral changes in centre of pressure (COP) displacements). Some of these findings were probably the result of head positioning and reduction of speed due to sedation, therefore to evaluate its clinical relevance this study should be repeated in patients with neurological disease to investigate naturally occurring neurological gait disturbances in horses [3].

Today, an increasing number of studies on equine gait are using quantitative measurement systems to document or support their findings. Nowadays, it is expected that clinical studies on lameness use objective measures for lameness quantification in combination with visual lameness assessment, and in some cases, objective recording of conformation and standing posture, not only because of the high inter-rater disagreement that exists, particularly for hindlimb lameness, but also because quantitative description of the gait during lameness can be statistically tested. Equipment like force plates and 
optic motion capture systems, the gold standard within objective gait technologies, are mainly restricted to research investigations in laboratory environments, being less accessible and less practical for clinical use. However, some private and University hospitals are using these types of equipment to document treatment outcomes for clinical research purposes as well as for clinical evaluations. An advantage of kinetic analysis using force plates is that ground reaction forces are more directly affected by changes in the limb loading capacity, opposed to changes in motion asymmetry during lameness which is an indirect measurement of lameness [1]. In the absence of force plate instrumentation, a simple method for equine kinematic gait event detection has been developed, which identifies the timing of hoof contact (hoof-on), peak vertical force and lift off (hoof-off) during walk, trot and canter. Identifying the moment in the stride when the peak vertical force occurs can be useful for lameness evaluation because it has been associated with higher risk for musculoskeletal injury [4]. On the other hand, an advantage of motion capture systems is that they can provide a detailed description of angular rotations and displacements of any body part, including head, pelvis, spine or limbs. Another alternative gait data quantification are foot pressure systems, which can give almost instantaneous analysis of limb loading including details of intra-hoof parameters. One disadvantage worth mentioning of these systems is that they do not provide data on acceleration and deceleration forces, (available with force plate measurement systems), but this limitation is outweighed by the detailed description that they can provide. This is demonstrated in a study using pressure plate measurements to investigate the development of hoof balance and landing preference in the post-natal period [5]. The study showed that new-born foals have more variable dynamic hoof balance, less oriented towards the lateral side of the hoof and to the heel than in mature horses, and gradually changing in the first weeks of life. Another study of the same authors aimed at quantifying gait kinetics in foals during the first half year of life, taking into account their osteochondrosis status. This showed that during growth, velocity at walk and trot gradually increases, while maintaining normalised peak vertical force; and that a temporary reduction in normalised peak vertical force was detected in osteochondrosis positive foals in the absence of lameness [6]. These findings in young horses should be taken into account when foals are presented with gait disturbances.

Inertial measurement units (IMUs) are wireless, smaller and cheaper alternative for lameness quantification based on motion asymmetry analysis. They are accessible and can be used quickly and are therefore very applicable to clinical practice. They are commonly used to confirm the lame limb in subtle lameness, thus may reducing time spent on evaluation of lame horses, to help less experienced clinicians to gain confidence and experience, and to distinguish push-off from impact types of lameness. Having an objective measure of asymmetry offers a quantitative tool to help evaluating the effects of any treatment, especially when the same clinician is not available to examine a horse in different occasions. However, IMUs are simpler than other systems and use very few gait parameters compared to visual examination. Although, this can be seen as a disadvantage, the simplicity and practicality of IMUs make them more popular for lameness evaluation than any other gait analysis system. IMUs provide measurements of vertical displacement asymmetry of the upper body (e.g. head nod and pelvic rise). Moreover, recent research into vertical displacement and asymmetry at the withers is looking promising to distinguish primary from secondary lameness. One study has shown that the vertical asymmetry pattern of the withers discriminated head nod of true forelimb lameness from the compensatory head motion asymmetry caused by primary hindlimb lameness [7]. Further research showed that the direction of the head versus the withers movement asymmetry identifies $69-77 \%$ of horses with ipsilateral and contralateral head and pelvic movement asymmetries [8].

There are several commercial IMU systems for horses available currently, most of them based on the asymmetry of the upper body, but some are looking at the motion asymmetry of the limbs instead of relying only in the changes of movement of head and pelvis, thus considering changes in the stride 
events. A study using distal limb mounted IMU sensors determined preliminary temporal kinematic stride variables at walk and trot [9]. Further investigation should be performed to improve the precision of this method for lameness diagnosis in clinical cases.

One disadvantage of using IMUs or any marker-based motion analysis is that markers need to be placed over specific anatomical locations and great care should be taken when positioning the sensors or markers, particularly when placed by different users. Sensors misplacement significantly affects the results of symmetry of the pelvis [10]. Therefore accurate information on anatomical placement of devices should be available for clinicians wanting to use objective gait analysis, as with any other type of equipment or technology. Another study investigated the use of a standalone consumer grade smartphone and compared it to a validated inertial measurement unit for quantification of movement symmetry. The smartphone provided meaningful data, however the placement of the device also affected the measurements [11]. Biplane high-speed fluoroscopy is a gait analysis technology that does not rely on sensor placement on specific anatomical locations and is not affected by skin displacement. This technique been used to study distal limb kinematics and to validate two different tracking possibilities [12]. Such technologies are out of reach of the equine practitioner at the moment, but can certainly contribute to the understanding of tri-dimensional rotations of the distal limb in normal and lame horses.

IMUs have also been recently used for gait analysis in Thoroughbreds, establishing gait parameters by performing a repeatability study [13] and investigating natural gait asymmetries in this type of horses, specifically studying the relation between head and withers movement asymmetry during lameness [8.] Another use of IMUs has been demonstrated in a study investigating the rehabilitation of spinal muscle groups affected by asymmetrical loading of the vertebral column due to lameness, after this has been resolved by veterinary intervention. The study investigated the effect of a 4-week elastic resistance band training regimen on back kinematics to promote muscular rehabilitation and development in horses. Results suggest that the elastic bands reduce thoracolumbar motion, thus increasing dynamic stability [14]. Studies like this one are examples of the use of objective gait data to support or dismiss alternative treatments, complementary therapies or any exercise programme claiming a particular effect on gait or standing posture symmetry.

Finally, wireless activity monitoring systems are increasingly becoming popular in humans and animals. A dual-mode accelerometer-based behavioural activity monitor was used to quantify locomotor activity in horses, with a step-counting function. The device identified different levels of activity and different gaits accurately, particularly when positioned in the hindlimb [15]. In can be foreseen that in the not too distant future, activity monitoring in horses, automated lameness monitoring and prevention and integrated biofeedback for rider/rider unit will be widely available.

In conclusion, plenty has been learnt with respect to equine lameness through biomechanic analysis, however we need to continue to investigate it to further elucidate the details of the mechanisms of lameness compensation, the biological significance of different thresholds of asymmetry under circumstances other than trotting in straight line, to distinguish and quantify proximal from distal lameness locations through objective gait analysis, and to improve the integration of biomechanic gait analysis with imaging and clinical evaluation for lameness diagnosis.

\section{B. Gómez Álvarez}

School of Veterinary Medicine, University of Surrey, Guildford, UK

Email: Constanza@evj.co.uk 


\section{References}

1. van Weeren, PR, Pfau, T, Rhodin, M, Roepstorff, L, Serra Bragança, FM, Weishaupt, MA. 2017. Do we have to redefine lameness in the era of quantitative gait analysis?. Equine Vet. J. 49, 567-569.

2. van Weeren, PR, Pfau, T, Rhodin, M, Roepstorff, L, Serra Bragança, FM, Weishaupt, MA. 2018. What is lameness and what (or who) is the gold standard to detect it. Equine Vet. J. 50, 549-551.

3. Nout-Lomas, YS, Page, KM, Kang, HG, Aanstoos, ME, Greene, HM. 2017. Objective assessment of gait in xylazine-induced ataxic horses. Equine Vet. J. 49, 3, 334-340.

4. Holt, D, St George, LB, Clayton, HM, Hobbs, SJ. 2017. A simple method for equine kinematic gait event detection. Equine Vet. J. 49, 334-340.

5. Gorissen, BMC, Serra Bragança, FM, Wolschrijn, CF, Back, W, van Weeren, PR. 2018. The development of hoof balance and landing preference in the postnatal period. Equine Vet. J. $50,809-817$.

6. Gorissen, BMC, Wolschrijn, CF, Serra Bragança, FM, Geerts, AAJ, Leenders, WOLJ, Back, W, van Weeren, PR. 2017. The development of locomotor kinetics in the foal and the effect of osteochondrosis. Equine Vet. J. 49, 467-474.

7. Rhodin, M, Persson-Sjodin, E, Egenvall, A, Serra Bragança, FM, Pfau, T, Roepstorff, L, Weishaupt, MA, Thomsen, MH, van Weeren, PR, Hernlund, E. 2018. Vertical movement symmetry of the withers in horses with induced forelimb and hindlimb lameness at trot. Equine Vet. J. 50, 818-824.

8. Pfau, T, Noorwijk, K, Sepulveda Caviedes, MF, Persson-Sjodin, E, Barstow, A, Forbes, B, Rhodin, M. 2018. Head, withers and pelvic movement asymmetry and their relative timing in trot in racing Thoroughbreds in training. Equine Vet. J. 50, 1, 117-124.

9. Serra Bragança, FM, Bosch, S, Voskamp, JP, Marin-Perianu, M, Van der Zwaag, BJ, Vernooij, JCM, van Weeren, PR, Back, W. 2017. Validation of distal limb mounted inertialmeasurement-unit sensors for stride detection in Warmblood horses at walk and trot. Equine Vet. J. 49, 4, 545-551.

10. Serra Bragança, FM, Rhodin, M, Wiestner, T, Hernlund, E, Pfau, T, van Weeren, PR, Weishaupt, MA. 2018. Quantification of the effect of instrumentation error in objective gait assessment in the horse on hindlimb symmetry parameters. Equine Vet. J. 50, 3, 370-376.

11. Pfau, T and Weller, R. 2017. Comparison of a standalone consumer grade smartphone to a specialist inertial measurement unit for quantification of movement symmetry in the trotting horse. Equine Vet. J. 49, 1, 124-129.

12. Geiger, SM, Reich, E, Böttcher, P, Grund, S, Hagen, J. 2018. Validation of biplane high-speed fluoroscopy combined with two different non-invasive tracking methodologies for measuring in vivo distal limb kinematics of the horse. Equine Vet. J. 50, 2, 261-269.

13. Sepulveda Caviedes, MF, Forbes, BS, Pfau, T. 2018. Repeatability of gait analysis measurements in Thoroughbreds in training. Equine Vet. J. 50, 4, 513-518.

14. Pfau, T, Simons, V, Rombach, N, Stubbs, N, Weller, R. 2017. Effect of a 4-week elastic resistance band training regimen on back kinematics in horses trotting in-hand and on the lunge. Equine Vet. J. 49, 6, 829-835.

15. Fries, M, Montavon, S, Spadavecchia, C and Levionnois, OL. 2017. Evaluation of a wireless activity monitoring system to quantify locomotor activity of horses in experimental settings. Equine Vet. J. 49, 2, 225-231. 